\title{
Improved third-generation lentiviral packaging with pLKO.1C vectors
}

\author{
Sunhye Lee ${ }^{1,2} \&$ David Cobrinik*,1,2,3,4,5
}

\begin{abstract}
A widely used third-generation lentiviral packaging system produces virus with enhanced biosafety by eliminating HIV accessory genes and separating packaging elements into three different plasmids. However, for certain vectors such as pLKO.1, third-generation safety features reduce lentiviral titers due to the lack of the accessory gene tat. Here we present a way to improve virus production and target gene knockdown with a modified pLKO.1 CMV pLKO.1C) vector and optimized packaging construct ratios. Replacing the pLKO.1 RSV promoter with the Cytomegalovirus promoter yielded an average of threefold higher titer than standard pLKO.1 packaged using the third-generation system, while optimizing the packaging vector ratios further increased titer and yielded an average of tenfold higher titer than PLKO.1 packaged with the second-generation system.
\end{abstract}

\section{METHOD SUMMARY}

Substituting the Rous Sarcoma Virus promoter of pLKO.1 with the Cytomegalovirus promoter dramatically enhanced virus production with the third-generation packaging system. Higher titers and improved target gene knockdown were achieved by optimizing the ratio of viral packaging constructs. This study suggests an approach to generate and deliver lentiviruses with maximized efficacy while maintaining biosafety.

\section{KEYWORDS}

lentivirus $\cdot$ pLKO.1 $\cdot$ shRNA $\cdot$ third-generation viral packaging

'The Vision Center, Children's Hospital Los Angeles, Los Angeles, CA 90027, USA; ${ }^{2}$ The Saban Research Institute, Children's Hospital Los Angeles, Los Angeles, CA 90027, USA; ${ }^{3}$ RoskiEye Institute\&Department of Ophthalmology, Keck School of Medicine of the University of Southern California, Los Angeles, CA 90033, USA; ${ }^{4}$ Department of Biochemistry \& Molecular Medicine, Keck School of Medicine of the University of Southern California, LoS Angeles, CA 90033, USA; ${ }^{5}$ Norris Comprehensive Cancer Center, Keck School of Medicine of the University of Southern California, Los Angeles, CA 90033, USA; *Author for correspondence: dcobrinik@chla.usc.edu

BioTechniques 68: 349-352 (June 2020) 10.2144/ btn-2019-0155
Lentiviral vectors are efficient tools for introducing genes into nondividing as well as dividing cells [1,2]. The most widely used lentiviral vectors are based on HIV but have been extensively modified to achieve high virus titers and decrease the risk of producing replication-competent virus [3-11]. To maximize expression of vector RNAs in virus-producing cells, most lentiviral vectors employ a chimeric promoter containing a strong RNA pol II promoter that initiates transcription at the first base of the long-terminal repeat (LTR) ' $R$ ' element. To improve biosafety, lentiviral vectors retain all cis-acting sequences needed for viral RNA packaging, reverse transcription and proviral DNA integration, yet remove all HIV protein-coding genes. HIV genes that are needed to produce infectious but nonreplication-competent virus are expressed separately, in trans, in lentiviral packaging cells [2].

Differences in lentiviral vectors and packaging systems can markedly affect virus production. For example, among the nine HIV protein-encoding genes, a widely used third-generation viral packaging system provides only gag, pol, rev and the VSV-G membrane fusogen, whereas the preceding second-generation system also expresses tat $[4,5]$. Tat protein is required for expression of lentiviral vectors that use the HIV LTR promoter but is not essential for Tat-independent chimeric promoters such as the immediate-early Cytomegalovirus (CMV) or Rous Sarcoma Virus (RSV) promoters $[4,8]$. However, Tat may increase vector expression by enabling transcriptional elongation through the TAR element in the ' $R$ ' component of the HIV 5' LTR [12,13].

pLKO.1 is one of the most widely used lentiviral vectors for shRNA expression due to its transduction efficacy, low levels of recombination, high-level shRNA expression and use in shRNA libraries [14]. As a thirdgeneration self-inactivating lentiviral vector, pLKO.1 contains a hybrid RSV promoter and HIV 5' LTR and is amenable to both second- and thirdgeneration packaging. However, at previously defined packaging vector ratios, pLKO.1 produces lower titers using the third-generation packaging system compared with the secondgeneration system. Since lentiviral vectors containing the hybrid CMV-HIV promoter often yield high lentiviral vector expression [15,16], we examined whether substituting the CMV promoter for the pLKO.1 RSV promoter could improve viral titer.

First, we cloned the CMV promoter from MA1 [16; originally derived from pCCL.sin.cPPT.PGK.GFP.WPRE [7]] into pLKO.1 [14; Addgene] or into various pLKO.1 derivatives to generate pLKO.1C vectors (Figure $1 \mathrm{~A} \& \mathrm{~B}$ ).

To test the activity of each promoter and packing system, we produced virus by reverse transfection of 293T cells using the indicated vector and packaging constructs as described [17]. For each 15-cm dish, a total of $50 \mu \mathrm{g}$ of plasmid DNA was used for the transfection for second-generation packaging (20 $\mu \mathrm{g}$ pLKO-based transfer vector, $20 \mu \mathrm{g}$ pCMV-dR8.91, $10 \mu \mathrm{g}$ pVSV-G), and $70 \mu \mathrm{g}$ of total plasmid was used for third-generation packaging (20 $\mu \mathrm{g}$ pLKO-based transfer vector, $20 \mu \mathrm{g}$ pMDLg/pRRE, $10 \mu \mathrm{g}$ pVSV-G and $20 \mu \mathrm{g}$ pRSV-Rev) (Figure 1C). After $60 \mathrm{~h}$, viral supernatants were harvested and concentrated 76-fold by centrifugation [17]; resuspended viruses were used to transduce cell lines in the presence of polybrene.

In initial tests, pLKO.1-shSCR was produced using second- or thirdgeneration packaging, whereas 
pLKO.1C-shSCR was produced solely with the third-generation system (Figure 1C). After transduction, DAOY cells were selected with puromycin for 4 days, fixed and stained with crystal violet to visualize puromycin-resistant colonies. This revealed that third-generation packaging of pLKO.1 yielded lower viral titers than second-generation packaging, whereas third-generation packaging of pLKO.1C yielded a qualitatively higher titer (Figure 1D).

To titer the virus more accurately, we generated $\mathrm{pLKO}$.1C-YFP by substituting the CMV for RSV promoter in pLKO.1 derivative
pLKO.1-YFP [17]. DAOY cells were transduced with either pLKO.1-YFP (shSCR and shRB1-733) or the corresponding PLKO.1CYFP vectors, and the titers calculated after counting yellow fluorescent protein (YFP)positive cells by FACS. Similar to the previous result, pLKO.1-YFP constructs had reduced viral titer using third-generation versus second-generation packaging, whereas the CMV-driven pLKO.1C-YFP virus had more than three-times higher titer with the thirdgeneration system (Figure 1E).

Since different amounts of each lentiviral vector and packaging construct have been suggested [18], we compared different ratios of the four viral components to define the optimal conditions with pLKO.1C-shSCR vectors (Figure 2A). 293T cells were transfected with third-generation packaging constructs at four ratios (with $20 \mu \mathrm{g}$ lenti shRNA vector per $15-\mathrm{cm}$ dish), and $1-2 \%$ of harvested virus was used to monitor the transduction of DAOY cells by selection and crystal violet staining of puromycin-resistant cells. The highest titer was obtained using a 4:2:1:1 ratio of transfer vector plasmid (pLKO.1 or pLKO.1C), packaging plasmid (pMDLg/pRRE), VSV fusogen plasmid

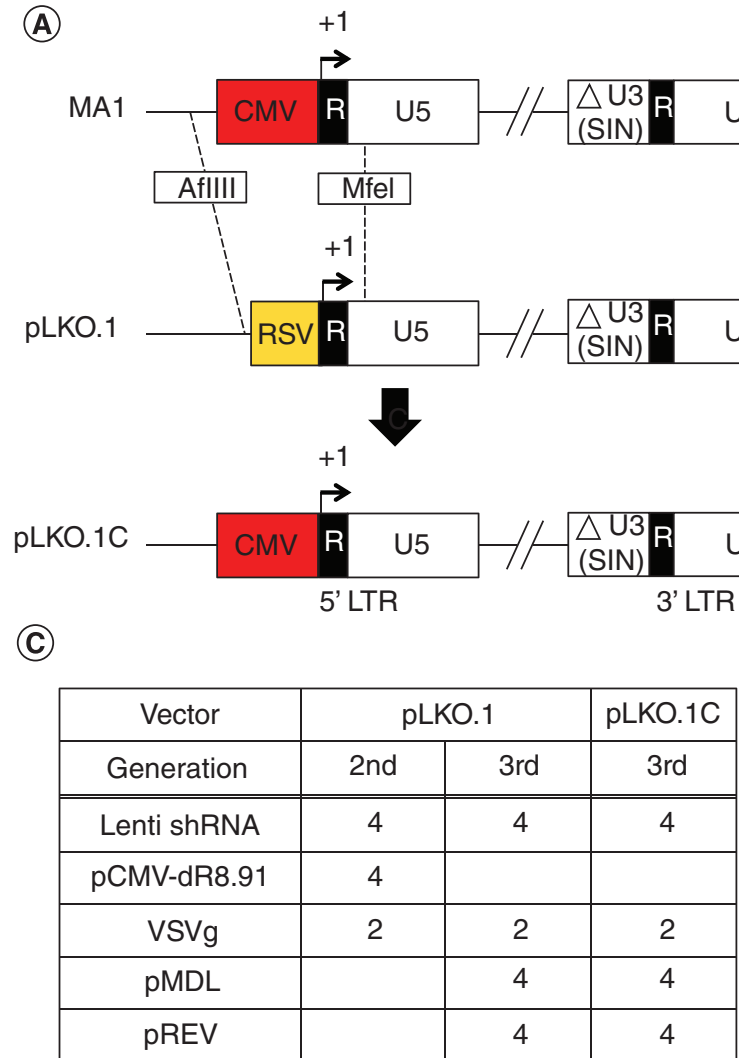

(D)

$\operatorname{shSCR}$

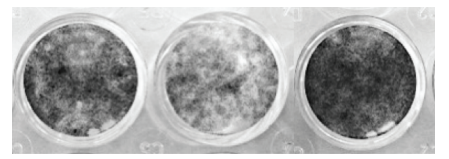

(B)
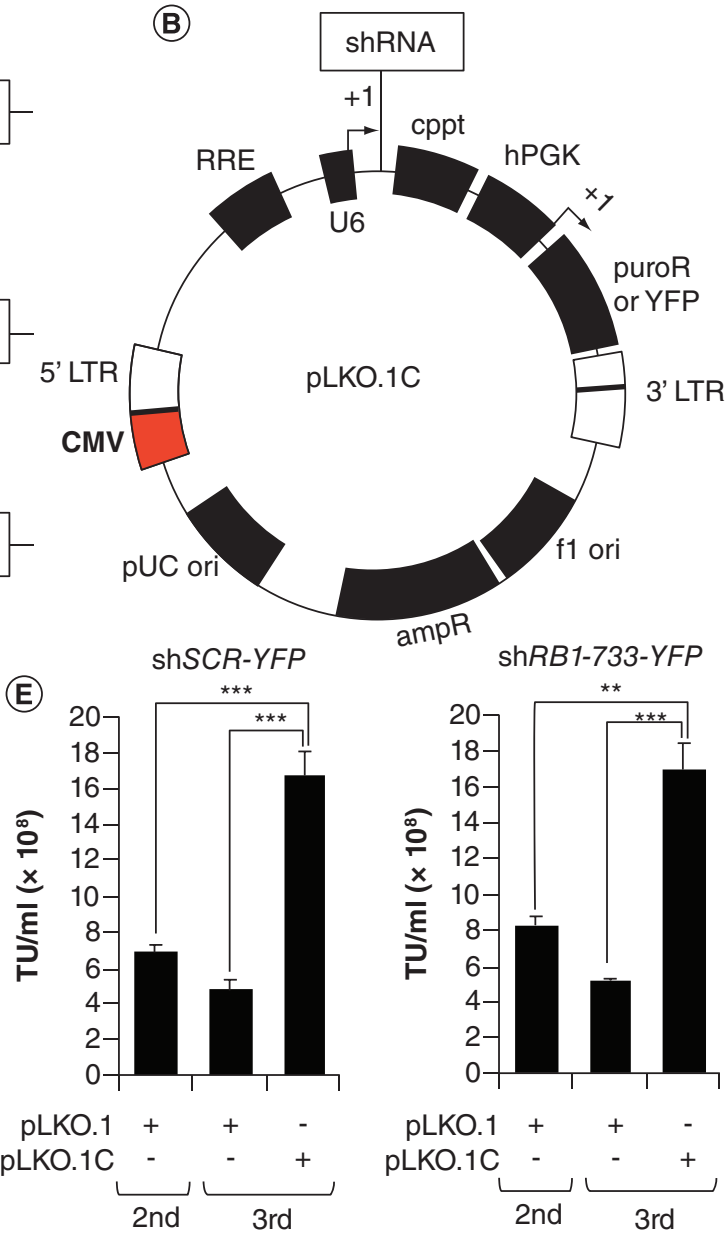

Figure 1. Improved transduction efficiency by pLKO.1C and third-generation lentiviral packaging. (A \& B) pLK0.1C cloning strategy. pLKO.1 and MA1 vectors were digested with Afllll and Mfel, and the 5.7-kb pLKO.1 vector and 1.8-kb CMV fragments were extracted and ligated. (C) Ratios of second- or third-generation lentiviral packaging plasmids for comparison of pLK0.1 versus $\mathrm{pLK0.1C}$ or $\mathrm{pLKO.1-YFP}$ versus $\mathrm{pLKO.1C-YFP} \mathrm{virus} \mathrm{production.} \mathrm{293T}$ cells were transfected with each ratio of lentiviral packaging plasmids and viruses were harvested after $60 \mathrm{~h}$. (D) DAOY cells transduced with pLKO.1 or pLKO.1C virus generated from the indicated viral packaging components and ratios in the presence of $4 \mu \mathrm{g} / \mathrm{ml}$ polybrene (Sigma-Aldrich), selected in $2 \mu \mathrm{g} / \mathrm{ml}$ puromycin and stained with crystal violet (Sigma-Aldrich) after 4 days. (E) DAOY cells transduced with virus carrying either shSCR (left) or shRB1-733 (right) generated with pLKO.1-YFP- or pLKO.1C-YFP-based vectors, with the viral packaging components and ratios shown in (C), followed by FACS to define the transforming units (TU) per $\mathrm{ml}$ of producer cell supernatant. $\mathrm{n}=2$ per group. Unpaired t-test. Experiments were performed at least twice with similar results.

$* * \mathrm{p}<0.02 ; * * * \mathrm{p}<0.01$.

CMV: Cytomegalovirus; YFP: Yellow fluorescent protein. 


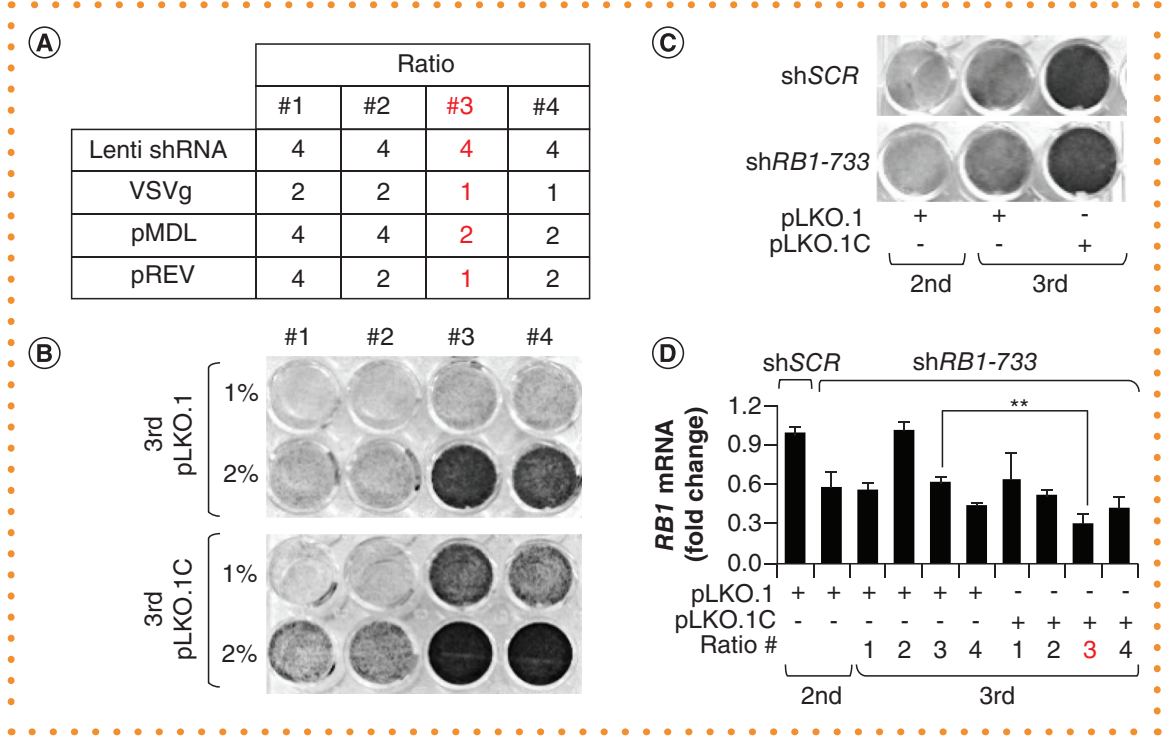

(B)
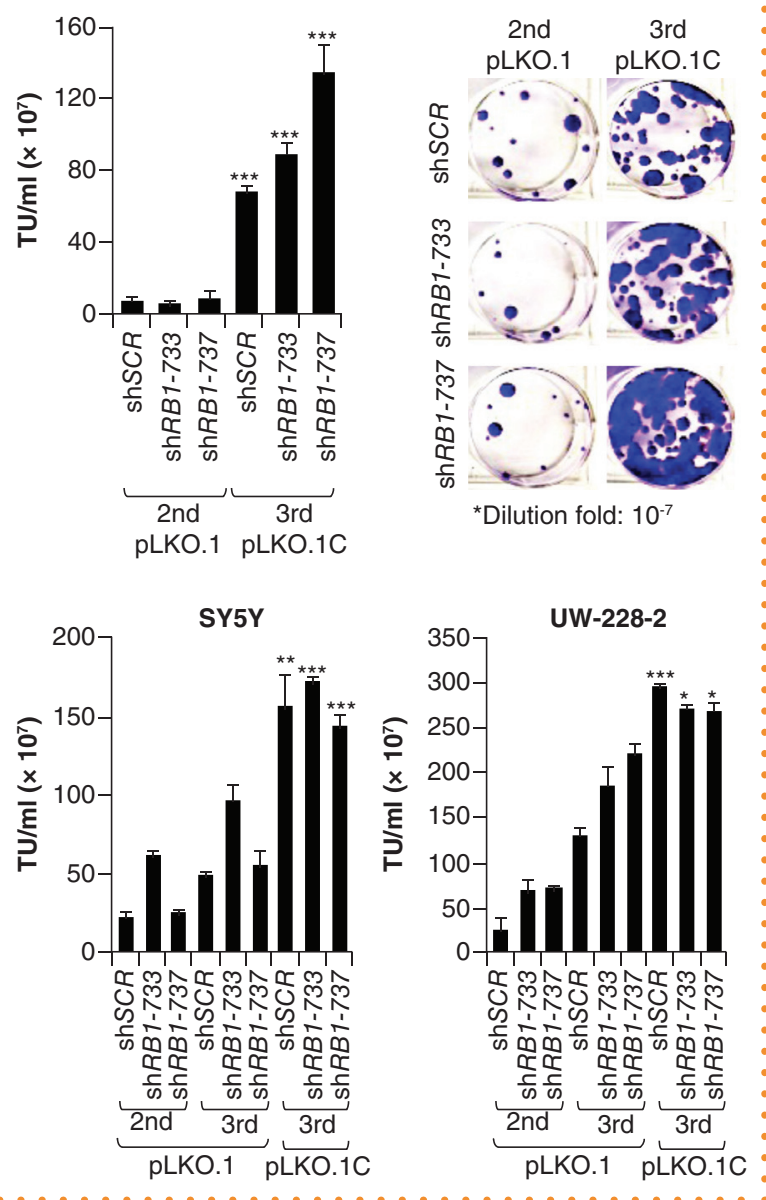

Figure 3. High viral titers produced from pLKO.1C with optimal ratio of viral components. (A-C) pLKO.1 and pLKO.1C produced with optimized viral packaging construct ratios were used to transduce DAOY cells with second- or third- generation packaging, followed by assessment of viral p24 antigen (A) and viral titers (B \& C) as described (Sigma Mission shRNA Manual). (D) Improved transduction efficiency of pLKO.1C evaluated with other cell lines. Values represent means of duplicate measurements and error bars the standard deviation. $\mathrm{n}=2$. Unpaired $\mathrm{t}$-test. ${ }^{*} \mathrm{p}<0.05 ;{ }^{* *} \mathrm{p}<0.02 ; * * * \mathrm{p}<0.01$.

Figure 2. Improved transduction by pLKO.1C at optimized viral component ratios. (A) Ratios of third-generation lentiviral packaging plasmids used for optimization. (B) DAOY cells transduced with 1 or $2 \%$ of total virus generated from different viral packaging ratios, puromycin-selected and visualized by crystal violet staining. (C) DAOY transduction with pLKO.1 (RSV) or pLKO.1C (CMV) versions of shSCR or shRB1-733 lentivirus after secondor optimized third-generation packaging, followed by puromycin selection and crystal violet staining. (D) DAOY transduction with the indicated vectors and ratios. After 4 days, RNA was isolated, and qRT-PCR was performed to measure RB knockdown efficacy. $n=3$. Unpaired t-test.

$\star \star p<0.02$.

CMV: Cytomegalovirus; RSV: Rous sarcoma virus.

(pVSV-G), and pRSV-Rev plasmid, respectively (Figure 2B). With these ratios, thirdgeneration packaging of $\mathrm{pLKO} .1 \mathrm{C}$ vectors gave higher efficiency than the secondgeneration packaging system (Figure 2C).

To examine whether the optimized virus packaging ratios improve target gene knockdown, we transduced cells with pLKO.1- and pLKO.1C-derived viruses carrying shRNA targeting RB1 (shRB1733). Viruses generated from different ratios of viral components, promoters and packaging systems (as in Figure 2A) were used to transduce DAOY cells and RB1 mRNA quantitated by qRT-PCR [17] at 4 days posttransduction. Consistent with the viral titering results, third-generation packaging of pLKO.1C with 4:2:1:1 ratio resulted in the best RB1 RNA knockdown (Figure 2D).

To quantitate the improved virus production and titers enabled by pLKO.1C, we produced $\mathrm{pLKO} .1$ and $\mathrm{pLKO} .1 \mathrm{C}$ versions of the shSCR, shRB1-733 and shRB1-737viruses using the optimized packaging ratios, and monitored viral p24 antigen concentration and viral titer by limiting dilution assay. After transduction of DAOY cells, twice as much p24 antigen and approximately ten-times higher virus titers were achieved with thirdgeneration packaging and pLKO.1C compared with second-generation packaging with pLKO.1 (Figure 3A-C). A similar higher transducing efficiency was observed with thirdgeneration packaging and $\mathrm{pLKO} .1 \mathrm{C}$ in several additional cell lines (Figure 3D). Although the ratios of shSCR, shRB1-733 and shRB1-737 titers varied, optimized third-generation 
packaging of pLKO.1C-shSCR consistently gave tenfold higher titers than second-generation packaging of pLKO.1. Taken together, introducing the CMV promoter into pLKO.1 greatly enhanced virus production, transduction and target gene knockdown using an optimized third-generation packaging system.

This study demonstrates a strategy to significantly improve lentiviral production with RSV-based lentiviral transfer vectors and third-generation viral packaging. The third-generation system expresses the minimal set of HIV-1 genes required to produce virus and omits tat because it is not required for promoter activity of transfer vectors that have chimeric CMV-HIV or RSV-HIV promoters $[4,8]$. However, removal of Tat may decrease lentiviral vector RNA expression by decreasing transcriptional elongation through the HIV TAR element [13]. The higher production of pLKO.1C virus may primarily relate to the CMV promoter's strength in 293T cells [19] as the hybrid CMV-HIV promoter (like the RSV-HIV promoter) is likely to remain partially Tat-dependent [20]. Our data reveal that the viral yield and transducing efficiency of the third-generation packaging system were significantly improved by replacing the RSV promoter of pLKO.1 with the CMV promoter and optimizing the ratio of viral packaging components. We suggest that this is a robust method to improve transduction efficiency while retaining the significant biosafety features of the third-generation lentiviral packaging system.

pLKO.1C (with stuffer and PuroR), pLKO.1C-YFP (with stuffer and YFP), pLKO.1C-shSCR and pLKO.1C-YFP-shSCR have been deposited at AddGene.

\section{AUTHOR CONTRIBUTIONS}

$S$ Lee designed and performed experiments and wrote the manuscript. D Cobrinik designed experiments and co-wrote the manuscript.

\section{FINANCIAL \& COMPETING INTERESTS DISCLOSURE}

This study was supported by the Margaret E Early Charitable Research Trust, an Alex's Lemonade Stand Foundation Young Investigator Award, and NIH grants R01CA137124 and R01EY0266610. The authors have no other relevant affiliations or financial involvement with any organization or entity with a financial interest in or financial conflict with the subject matter or materials discussed in the manuscript apart from those disclosed.

No writing assistance was utilized in the production of this manuscript.

\section{ACKNOWLEDGMENTS}

MA1 vector was kindly provided by $L$ Naldini of San Raffaele Telethon Institute for Gene Therapy. The authors thank D Shayler for contributing pLKO.1C-YFP.

\section{OPEN ACCESS}

This work is licensed under the AttributionNonCommercial-NoDerivatives 4.0 Unported License. To view a copy of this license, visit http://creativecommons.org/licenses/ by-nc-nd/4.0/

\section{REFERENCES}

1. Naldini $\mathrm{L}, \mathrm{Blomer} \mathrm{U}$, Gallay $\mathrm{P}$ et al. In vivo gene delivery and stable transduction of nondividing cells by a lentiviral vector. Science 272(5259), 263-267 (1996).

2. Naldini L, Trono D, Verma IM. Lentiviral vectors, two decades later. Science 353(6304), 1101-1102 (2016).

3. Zufferey R, Nagy D, Mandel RJ, Naldini L, Trono D. Multiply attenuated lentiviral vector achieves efficient gene delivery in vivo. Nat. Biotechnol. 15(9), 871-875 (1997).
4. Dull T, Zufferey R, Kelly $\mathrm{M}$ et al. A third-generation entivirus vector with a conditional packaging system. $J$. Virol. 72(11), 8463-8471 (1998).

5. Zufferey R, Dull T, Mandel RJ et al. Self-inactivating lentivirus vector for safe and efficient in vivo gene delivery. $J$. Virol. 72(12), 9873-9880 (1998).

6. Root DE, Hacohen N, Hahn WC, Lander ES, Sabatini DM. Genome-scale loss-of-function screening with a lentiviral RNAi library. Nat. Methods 3(9), 715-719 (2006).

7. Follenzi A, Ailles LE, Bakovic S, Geuna M, Naldini L. Gene transfer by lentiviral vectors is limited by nuclear translocation and rescued by HIV-1 pol sequences. Nat. Genet. 25(2), 217-222 (2000).

8. Kim VN, Mitrophanous K, Kingsman SM, Kingsman AJ. Minimal requirement for a lentivirus vector based on human immunodeficiency virus type 1. J. Virol. 72(1), 811-816 (1998)

9. Miyoshi H, Blomer U, Takahashi M, Gage FH, Verma IM. Development of a self-inactivating lentivirus vector. $J$. Virol. 72(10), 8150-8157 (1998).

10. Wagner R, Graf M, Bieler K et al. Rev-independent expression of synthetic gag-pol genes of human immunodeficiency virus type 1 and simian immunodeficiency virus: implications for the safety of lentiviral vectors. Hum. Gene Ther. 11(17), 2403-2413 (2000).

11. Gasmi M, Glynn J, Jin MJ, Jolly DJ, Yee JK, Chen ST. Requirements for efficient production and transduction of human immunodeficiency virus type 1-based vectors. J. Virol. 73(3), 1828-1834 (1999).

12. Schiralli Lester GM, Henderson AJ. Mechanisms of HIV transcriptional regulation and their contribution to latency. Biol. Int. 2012(614120) (2012).

13. Kao SY, Calman AF, Luciw PA, Peterlin BM. Anti-termination of transcription within the long terminal repeat of HIV-1 by tat gene product. Nature 330(6147), 489-493 (1987).

14. Moffat J, Grueneberg DA, Yang X et al. A lentiviral RNAi library for human and mouse genes applied to an arrayed viral high-content screen. Cell 124(6), 1283-1298 (2006).

15. Boshart M, Weber F, Jahn G, Dorsch-Häsler K, Fleckenstein $B$, Schaffner W. A very strong enhancer is located upstream of an immediate early gene of human cytomegalovirus. Cell 41(2), 521-530 (1985).

16. Amendola M, Venneri MA, Biffi A, Vigna E, Naldini L. Coordinate dual-gene transgenesis by lentiviral vectors carrying synthetic bidirectional promoters. Nat. Biotechnol. 23(1), 108-116 (2005).

17. $\mathrm{Xu} X L$, Singh $H P$, Wang $L$ et al. Rb suppresses human cone-precursor-derived retinoblastoma tumours. Nature 514(7522), 385-388 (2014)

18. Cribbs AP, Kennedy A, Gregory B, Brennan FM. Simplified production and concentration of lentiviral vectors to achieve high transduction in primary human T cells. BMC Biotechnol. 13, 98 (2013).

19. Qin JY, Zhang L, Clift KL et al. Systematic comparison of constitutive promoters and the doxycycline-inducible promoter. PLOS ONE 5(5), e10611 (2010).

20. Robinson D, Elliott JF, Chang LJ. Retroviral vector with a CMV-IE/HIV-TAR hybrid LTR gives high basal expression levels and is up-regulated by HIV-1 Tat. Gene Ther. 2(4), 269-278 (1995). 